\title{
ARTICLE
}

\section{Functional near-infrared spectroscopy in psychiatry}

\author{
Cynthia Y. Y. Lai, Cyrus S. H. Ho, Charmaine R. Lim \& Roger C. M. Ho
}

Cynthia Lai is an Assistant Professor in the Department of Rehabilitation Sciences at the Hong Kong Polytechnic University, and an occupational therapist who specialises in paediatrics and working with people with developmental disabilities. Cyrus $\mathbf{H o}$ is an associate consultant psychiatrist in the Department of Psychological Medicine, National University Hospital, Singapore. He has a special interest in functional imaging. Charmaine Lim is a doctoral candidate in clinical psychology at James Cook University, Singapore. She is currently training as a clinical psychologist and is a Member of the Singapore Psychological Society.

Roger Ho is an Associate Professor and Consultant Psychiatrist in the Department of Psychological Medicine, National University of Singapore. He is a Member of the Royal College of Psychiatrists and Fellow of the Royal College of Physicians (Canada). He was conferred MD (by research) by the University of Hong Kong. Correspondence Roger C. M. $\mathrm{Ho}$, Department of Psychological Medicine, National University of Singapore, Level 9 NUHS Tower Block, 1E Kent Ridge Road Singapore. Email: roger_ho@nuhs. edu.sg

\section{Copyright and usage}

(C) The Royal College of Psychiatrists 2017.

\section{SUMMARY}

Functional near-infrared spectroscopy (fNIRS) has been used in healthcare and medical research for the past two decades. In particular, the use of fNIRS in academic and clinical psychiatry has increased rapidly owing to its advantages over other neuroimaging modalities. fNIRS is a tool that can potentially supplement clinical interviews and mental state examinations to establish a psychiatric diagnosis and monitor treatment progress. This article provides a review of the theoretical background of fNIRS, key principles of its applications in psychiatry and its limitations, and shares a vision of its future applicability in psychiatric research and clinical practice.

\section{LEARNING OBJECTIVES}

- Understand the theoretical background, mechanism of action and clinical applications of fNIRS and compare it to other neuroimaging modalities

- Understand the use of fNIRS in academic and clinical psychiatry through current research findings

- Be able to evaluate the future potential of fNIRS and formulate new ideas for using fNIRS in academic and clinical psychiatry

\section{DECLARATION OF INTEREST}

None

Near-infrared spectroscopy (NIRS) has been used in medical research for more than 20 years. Based on the principles of NIRS, functional nearinfrared spectroscopy (fNIRS) is a non-invasive neuroimaging technology that maps the functioning of the cerebral cortex by measuring haemodynamics (Ferrari 2012). Although fNIRS technology was originally used in basic neuroscience research to investigate the motor, visual, language and executive functions of healthy participants, it is increasingly been used to study a wide range of psychiatric disorders, including schizophrenia (Ehlis 2007), depression (Fukuda 2012; Liu 2014; Takizawa 2014), bipolar disorder (Takizawa 2014), panic disorder (Nishimura 2009), obsessivecompulsive disorder (Okada 2013), addiction (Dempsey 2015), Alzheimer's disease (Chou 2013), autism spectrum disorder (Fekete 2014; Jung 2016) and attention-deficit hyperactivity disorder (ADHD) (Ehlis 2008; Inoue 2012; Ishii-Takahashi 2014). Most studies have attempted to delineate objective and measurable haemodynamic changes in the cerebral cortex in relation to the psychopathology of these psychiatric disorders. Hence, incorporating fNIRS into psychiatry may address the current limitations of clinical interviews and mental state examinations in establishing a psychiatric diagnosis.

Martinelli \& Shergill (2015) published a review about neuroimaging and psychiatry in BJPsych Advances. The review discussed conventional neuroimaging techniques using large, costly and immobile imaging devices, such as computed tomography (CT) scanning, magnetic resonance imaging (MRI), magnetic resonance spectroscopy (MRS), positron emission tomography (PET) and magnetoencephalography (MEG). These techniques require the participants to be in a supine position with their head restrained and without any movement of the body (Martinelli 2015). The scanning procedures are typically conducted by radiographers, with high costs incurred by each measurement. Although Martinelli \& Shergill described fNIRS as a tool primarily used with infants, its use has expanded to adult and elderly populations. The increasing popularity of fNIRS could be attributed to its advantages over conventional neuroimaging devices, i.e. that it:

- allows for measurements to be taken in any body position while retaining good temporal resolution

- has relatively low cost and volume

- does not require radiographers

- is compatible with a variety of neuroimaging instruments (Ferrari 2012).

Furthermore, an fNIRS device is highly portable owing to its light weight and small size, allowing measurements to be obtained in various clinical settings. Since fNIRS is not sensitive to body movement, it can also be used to measure 'fidgety' patients such as children with ADHD. Consequently, fNIRS has potential in assisting diagnosis, predicting clinical outcomes and 
evaluating treatment outcomes in psychiatry (Fukuda 2012; Koike 2013; Ho 2016). A limitation of fNIRS is its inability to detect haemodynamic changes in deep neuroanatomical structures (Chou 2013). Nevertheless, understanding the theoretical background of fNIRS as well as its strengths and limitations will facilitate further development of the technology.

The purpose of this article is to introduce the theoretical background of fNIRS, key principles in the application of fNIRS and its current use in various psychiatric disorders, and to share our vision of its future applications in academic and clinical psychiatry.

\section{Theoretical background of fNIRS}

\section{Brain-computer interface}

The fNIRS system is an example of a braincomputer interface. Each fNIRS device includes the following components:

- a set of optical fibres (NIR light emitter/detector)

- a computer unit with signal processor and algorithm

- a computer monitor (Fig. 1).

The NIR light emitter and detector probe come as a set. The probe set is positioned over different areas of the head, e.g. the prefrontal cortex, frontal lobe, parietal lobe, temporal lobe or pre-supplementary motor areas (Fig. 2). The positioning of fNIRS probes over the scalp is similar to the attachment of electroencephalogram (EEG) electrodes on the scalp according to the EEG

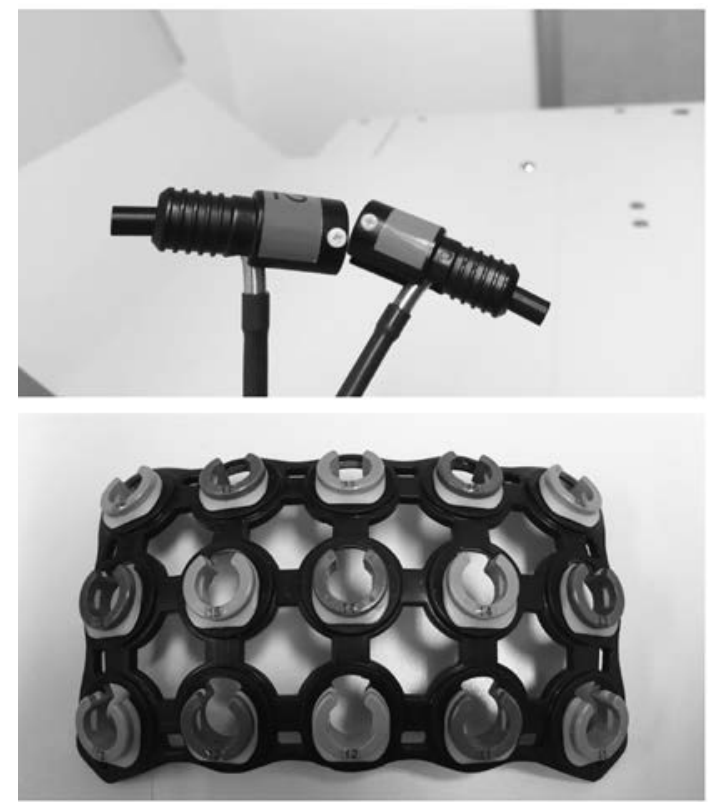

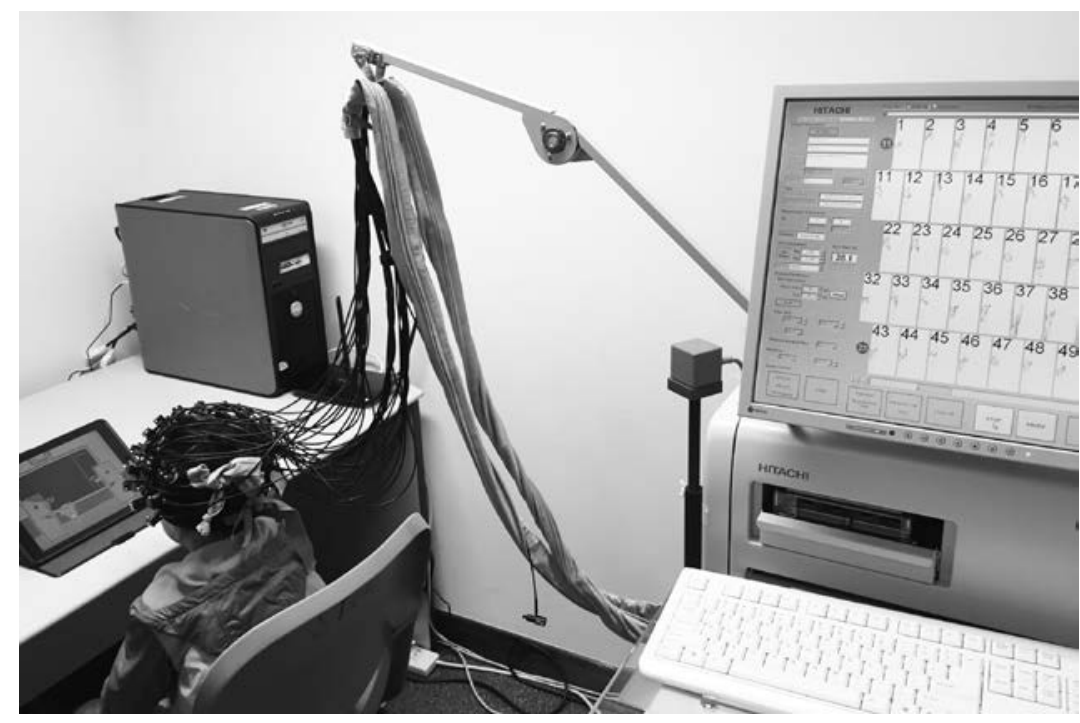

FIG 2 Data acquisition of cerebral haemodynamics during a tablet-based cognitive task.

10/20 system. A code is assigned to each probe for identification purposes. A topographical image of cerebral haemodynamics can be reconstructed and displayed on the monitor (online Fig. DS1). There are currently various different models of fNIRS devices, with some being particularly lightweight and designed for studying mobile participants, e.g. the NIRSport (online Fig. DS2).

\section{Brain activation}

Haemoglobin is a protein that forms an unstable and reversible bond to oxygen in red blood

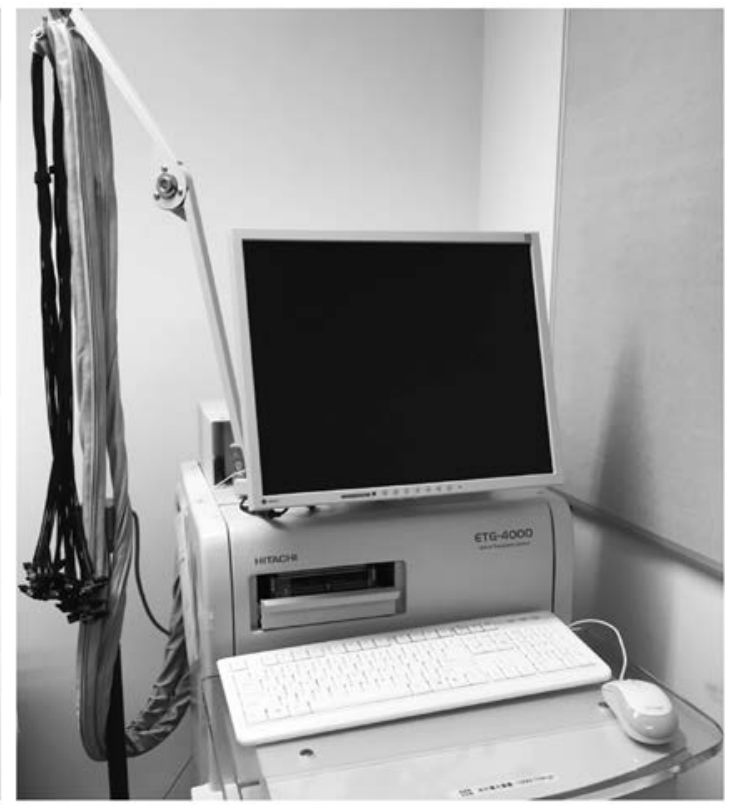

The components of a functional near-infrared spectroscopy (fNIRS) device: near-infrared light emitter/detector probes (upper-left), headgear to hold the probes (lower-left) and a computer unit with a signal processor (right). 
cells. In its oxygen-loaded form, it is bright red and known as oxyhaemoglobin (oxy-Hb). In its oxygen-unloaded form, it is purple/blue and known as deoxyhaemoglobin (deoxy-Hb). fNIRS can be used to examine haemodynamic activity in the brain by detecting simultaneous changes in the concentrations of oxy-Hb and deoxy$\mathrm{Hb}$. The rationale lies in a mechanism known as neurovascular coupling, whereby activation of a brain region is reflected by an increase in oxy-Hb and a corresponding decrease in deoxy$\mathrm{Hb}$ levels. When there is an increase in activity within a particular brain region, there would be an increase in blood supply and oxy-Hb levels. Although deoxy-Hb would also increase owing to oxygen consumption during the brain activity, this would be compensated for by the increase in blood supply, resulting in a net decrease in deoxy- $\mathrm{Hb}$ levels (Koike 2013).

Both fMRI and fNIRS map brain activity by measuring changes in haemoglobin concentration. Studies that have used fNIRS and fMRI concurrently to measure activity during cognitive tasks have found fNIRS results to be highly correlated $(r=0.77-0.94)$ with fMRI blood oxygen leveldependent (BOLD) signal recordings in the same neuroanatomical regions (e.g. Cui 2011). Hence, fNIRS can be an appropriate substitute for fMRI to study activities of the cerebral cortex when engaged in cognitive tasks. Researchers designing studies with fNIRS would need to consider:

- whether the spatial resolution of fNIRS is adequate to answer their question of interest

- whether their design accounts for weaker signalto-noise ratio, especially in brain regions more distal from the scalp (Cui 2011)

- that the fNIRS-Hb signal mainly reflects haemodynamic changes in the grey matter (Sato 2013).

\section{Nature of light}

fNIRS was developed based on the principles of NIR spectroscopy. According to Ferrari \& Quaresima (2012), human tissue is transparent to NIR light of wavelength $650-1000 \mathrm{~nm}$. This light can penetrate through the skin and bone with low absorption, to reach haemoglobin in the capillaries with high absorption (Koike 2013). The NIR light will be scattered in tissues or absorbed by chromophores. The fNIRS instrument utilises characteristics of light absorption by haemoglobin to assess changes in oxy-Hb and deoxy-Hb concentration in various brain regions.

The mechanism of fNIRS measurement involves the emission and absorption of light. Light emitted from the source probe (emitter) is scattered or absorbed. The scattered light will then be detected by detector probe located on the surface of the scalp. The source probe and detector probe are paired by modulation of light amplitude. The different light absorption rates of oxy-Hb and deoxy-Hb allow for the concentrations of oxy-Hb and deoxy$\mathrm{Hb}$ within a brain region to be measured. There are three different types of NIRS technique, each based on specific illumination methods:

- the continuous wave modality (providing constant tissue illumination)

- the frequency domain method (illuminating the head with intensity-modulated light)

- the time domain technique (illuminating the head with short pulses of light).

These three techniques are based on different characteristics of light emission and different detection mechanisms. Most commercial fNIRS tools adopt the continuous wave modality, which has the advantages of a higher sampling rate, relatively low cost and increased portability. However, the penetration power (depth of penetration) of continuous wave modality is lower than that of the frequency domain and time domain techniques. Furthermore, instruments using the continuous wave modality are only able to measure changes in levels of oxy-Hb and deoxy$\mathrm{Hb}$, whereas the other two techniques can measure absolute levels of oxy-Hb and deoxy-Hb (Ferrari 2012). Hence, the lack of absolute values of oxy$\mathrm{Hb}$ and deoxy-Hb concentration are a limitation of most commercial fNIRS devices.

fNIRS has several advantages over other neuroimaging tools. First, fNIRS is not sensitive to movement and can be used on fidgety patients, young children and participants performing either sitting- or movement-based tasks. Second, the light used in fNIRS does not interfere with other tools, such as fMRI, PET and EEG. Hence, multimodal measurements can be performed on patients (e.g. fNIRS and fMRI; fNIRS and EEG; or fNIRS and PET). Third, its source probe and detector probe can be paired and located to provide spatial information regarding the brain's topography, albeit with less satisfactory spatial resolution than fMRI. Fourth, the high speed of light and high sampling rate of current models of fNIRS device (0.1 s) may provide good temporal resolution, although the temporal resolution is still lower than that of EEG. Last, as some fNIRS devices are compatible with three-dimensional (3D) brain mapping software, a 3D image could be reconstructed with measurement data based on $3 \mathrm{D}$ coordinates. This can be done with or without importing fMRI measures and could certainly facilitate topographical representation of brain activation to assist in the diagnostic process. 
The fNIRS device has some inherent limitations owing to the characteristics of NIR light and its penetration and absorption. Brain activity measured by fNIRS is based on light absorption by haemoglobin in the blood. However, both brain activity and peripheral physiological changes may contribute to light absorption and influence measurement data. For instance, activation of the sympathetic and parasympathetic nervous systems may lead to vasodilation or vasoconstriction in the skin. It is challenging to differentiate taskinduced superficial haemodynamics from cerebral haemodynamics, even with steady blood flow to the skin (Takahashi 2011; Hoshi 2016), and in practice there will often be variation in the rate of blood flow across different regions of measurement within or between individuals. Skin blood flow artefacts can be removed by applying independent component analysis (ICA) if there is low correlation between skin blood flow and cerebral blood flow (Takanori 2011). Another limitation is the penetration power of light, which limits the depth of the brain region that can be reached; as a result, fNIRS may not be suitable for examining the activity of subcortical or deep brain structures (Chou 2013; Hoshi 2016). For example, the pathology of Alzheimer's disease involves deficits of deep brain structures, including the parahippocampal gyrus and hippocampus, which cannot be measured by fNIRS devices of continuous wave modality.

In summary, understanding the strengths and limitations of fNIRS may help in exploring its potential in psychiatric research and clinical practice.

\section{Principles of applying fNIRS}

Applying fNIRS to the diagnosis of psychiatric disorders and monitoring of treatment progress is a relatively new area that requires further development. When establishing a clinical diagnosis, hypotheses are first generated before being tested with appropriate methods. fNIRS could be considered as a potential aid to support the diagnosis of psychiatric conditions (Takizawa 2014). Here, we highlight some practical considerations in setting up the probes and designing paradigms, and refer to previous studies that illustrate key principles in applying fNIRS for psychiatric conditions.

\section{Single or multiple channels in measurement}

The emitter and detector probes of the fNIRS device are paired to form a channel over a defined measurement area. The number of probes can be customised according to the areas of interest. For example, a $3 \times 11$ grid will contain 52 measurement channels and a $4 \times 4$ grid will contain 24 . Research has used fNIRS with 2-channel (Kubota 2005), 22-channel (Ehlis 2007) and 52-channel systems (Yamanaka 2014). The advantages of using fewer channels include cost reduction, shortened preparation time and increased portability. When studying haemodynamics, the monitoring is limited to the cerebral cortex, which is a welldefined region suitable for study using a system with fewer channels. Otherwise, use of systems with fewer channels may run the risk of missing out important neuroanatomical areas (Ehlis 2007).

\section{Design of study paradigm}

In general, there are two types of design paradigm: block design and event-related design (also known as trial-based design). Block design examines the pattern of brain activation across conditions, such as an alternating pattern of one or more blocks of the resting condition and the task condition. The condition could also be set as a time course (e.g. the pre-task, during-task and after-task period). By contrast, event-related design examines brain activation triggered by events that occur in a random order rather than an alternating pattern. Event-related design offers greater flexibility and measures transient changes in brain activity over short and discrete intervals.

As the fNIRS device has good temporal resolution, it is suited to assessing brain activity through event-related design (Schroeter 2002). Depending on the brand and model of the fNIRS device, the person conducting the examination can either set their own protocol (with or without synchronising with a task created by computerised stimulus-presentation software) or apply built-in study paradigms as appropriate.

\section{Selecting brain areas and regions of interest}

Understanding neural correlates is fundamental in fNIRS. Currently, the diagnosis of psychiatric conditions relies on mapping findings from clinical presentation and mental state examination on to predefined diagnostic criteria in the DSM- 5 or ICD10. This process can be subjective and qualitative, and it does not elucidate the underlying pathology of a psychiatric condition. The symptoms and behaviours may be caused by particular activation and deactivation patterns of the cerebral cortex when engaged in cognitive tasks. Given that brain activity over particular brain areas can be characterised and measured using fNIRS, it is necessary to define respective brain areas (regions of interest) and brain functions for investigation.

Some psychiatric illnesses were found to be correlated with dysfunctions of specific neuroanatomical 
areas. For instance, schizophrenia has been associated with frontal cortex dysfunction, and taskrelated brain activation was hypothesised to differ between patients with schizophrenia and healthy controls. Ehlis et al (2007) examined frontal lobe activity in 12 patients with schizophrenia and 12 healthy controls, using fNIRS and a verbal fluency task (VFT). They found that frontal activation was significantly reduced in people with schizophrenia, compared with controls. Frontal lobe abnormalities, especially a decrease in bilateral frontotemporal oxygenation, have also been found in individuals with unipolar depression, using the VFT (Noda 2012). Online Fig. DS3 illustrates the differences in activation patterns during the VFT between a healthy control and a patient with depression who had received electroconvulsive therapy (ECT). Prefrontal cortex dysfunction was also found among adults with ADHD. Ehlis et al (2008) examined 13 adults with ADHD and 13 healthy controls by pairing fNIRS with a working memory task intended to assess prefrontal cortex function. The results showed that prefrontal activation was significantly lower in those with ADHD relative to controls. Inoue et al (2012) examined the haemodynamic response of the prefrontal cortex using fNIRS and the 'go/ no go' task. Individuals with ADHD demonstrated lower levels of brain activation in the prefrontal cortex compared with healthy controls during the inhibition response condition (no go condition), but not the execution response condition (go condition).

TABLE 1 Studies using functional near-infrared spectroscopy to assess different psychiatric disorders

\begin{tabular}{|c|c|c|}
\hline Study & Disorder & Key findings \\
\hline Ehlis et al, 2007 & Schizophrenia & $\begin{array}{l}\text { Verbal fluency tasks generally led to obvious frontal } \\
\text { activation in healthy controls and significantly reduced } \\
\text { frontal activation in patients with schizophrenia }\end{array}$ \\
\hline Ehlis et al, 2008 & $\begin{array}{l}\text { Attention-deficit } \\
\text { hyperactivity } \\
\text { disorder (ADHD) }\end{array}$ & $\begin{array}{l}\text { During the working memory ( } n \text {-back) task, individuals } \\
\text { with ADHD showed reduced task-related increases in } \\
\text { the concentration of oxyhaemoglobin (oxy-Hb) in the } \\
\text { ventro-lateral prefrontal cortex (PFC) }\end{array}$ \\
\hline Nishimura et al, 2009 & Panic disorder & $\begin{array}{l}\mathrm{Oxy}-\mathrm{Hb} \text { changes in the left inferior PFC were } \\
\text { significantly associated with frequency of panic attacks, } \\
\text { whereas deoxy-Hb changes in the anterior area of the } \\
\text { right PFC were significantly associated with severity of } \\
\text { agoraphobia }\end{array}$ \\
\hline Okada et al, 2013 & $\begin{array}{l}\text { Obsessive- } \\
\text { compulsive } \\
\text { disorder (OCD) }\end{array}$ & $\begin{array}{l}\text { During the Stroop colour-word task, oxy-Hb changes in } \\
\text { the PFC of patients with OCD were significantly smaller } \\
\text { than in healthy controls }\end{array}$ \\
\hline Liu et al, 2014 & Depression & $\begin{array}{l}\text { During the verbal fluency task, hypoactivation in lateral } \\
\text { and lower PFC was observed in patients with major } \\
\text { depressive disorder. Correlation analysis found that } \\
\text { oxy-Hb concentration exchange in the bilateral PFC } \\
\text { and anteromedial PFC were associated with severity of } \\
\text { depression }\end{array}$ \\
\hline
\end{tabular}

The above examples illustrate the feasibility of using fNIRS to identify unique brain activation patterns present among psychiatric patients by pairing it with activation tasks targeting specific cognitive processes. Table 1 summarises the key findings of studies using fNIRS to assess different psychiatric disorders. These studies were identified by keyword searches (for 'fNIRS' and 'specific psychiatric disorders') in PubMed.

\section{Differential diagnosis of psychiatric disorders}

Patients with different psychiatric illnesses may present with similar clinical features, increasing the challenge of diagnosis. It is possible that their symptomatology is related to dysfunction of particular neuroanatomical areas or reflected by distinct brain activity patterns. In 2014, Takizawa et al (2014) conducted a large-scale, multisite case-control study of group comparisons. A total of 673 patients with psychiatric disorders (major depressive disorder, bipolar disorder and schizophrenia with depressive symptoms) and 1007 healthy volunteers were examined. The study used 52-channel fNIRS and the VFT to measure relative oxy- $\mathrm{Hb}$ and deoxy- $\mathrm{Hb}$ signal changes in two brain regions: (a) frontopolar and dorsolateral prefrontal cortical regions; (b) the ventrolateral prefrontal cortex, and superior and middle temporal cortical regions. The fNIRS signal changes were measured over three periods - pre-task (baseline), task activation, and posttask - for a total of 125 seconds. This revealed that the centroid value (measured in seconds, and indicated by the position along the time course of a perpendicular line from the centroid of positive change of the NIRS signal area) in region 1 was able to correctly differentiate $74.6 \%$ of patients with major depressive disorder from healthy controls with no depression. The promise of fNIRS in assisting psychiatric diagnosis led the Japanese Health Ministry to approve fNIRS as an 'advanced medical technology' in 2014, and it is an insurance-covered investigation to aid differential diagnosis of depressive state (Fukuda 2015). There are currently no such initiatives in other countries.

The study by Takizawa et al (2014) provides insight into the applicability of fNIRS in differential diagnosis of psychiatric disorders and the feasibility of providing visual evaluation results to patients and caregivers. There is a need to develop a database of cerebral haemodynamic patterns of various psychiatric disorders to serve as a reference for future comparisons. fNIRS can augment clinical interviews and mental state examination in establishing a clinical diagnosis. It is possible that clinical symptoms in psychiatric 
disorders are secondary to low/exaggerated cortical or subcortical function together with suboptimal brain arousal state and other physiological dysregulation (e.g. autonomic dysfunction). As fNIRS can be simultaneously combined with other devices, future diagnostic approaches could involve multimodal measures (measuring other biological signals, such as EEG waves and autonomic activity) as a diagnostic tool in clinical psychiatry. Although fNIRS cannot produce detailed pictures of neuroanatomical structures, and hence cannot replace structural MRI, its portability and costeffectiveness support it as a potential diagnostic tool to verify fMRI findings (e.g. functional hypofrontality in schizophrenia). Nevertheless, it needs to be emphasised that although fNIRS has the potential to be a supplementary diagnostic tool, it cannot replace conventional history-taking and mental state examination in establishing a psychiatric diagnosis. In the near future, more assessment paradigms need to be developed and training in clinical application of fNIRS should be offered to psychiatrists.

\section{Conclusions}

This article provides a brief review of the theoretical background and principles of applying fNIRS in psychiatric research. fNIRS is a tool that can potentially facilitate the clinical diagnostic process and identify stages of psychiatric illnesses by providing objective and quantifiable evidences of brain changes (Nishimura 2009; Fukuda 2012). However, this will require specific cerebral haemodynamic patterns to be validated in larger clinical populations with specific psychiatric disorders. Large-scale studies are now possible, owing to advances in the fNIRS technology and improvements in its measurement accuracy. Additionally, it will be important to develop standardised paradigm protocols and data analysis methodologies to ensure reproducibility of results. As recommended by Koike (2013), establishing an international community of NIRS researchers may promote more active use of fNIRS and advance expertise in this challenging area. This will, in turn, shape developments in the role of fNIRS in complementing psychiatric diagnosis and management in years to come.

\section{References}

Chou PH, Lan TH (2013) The role of near-infrared spectroscopy in Alzheimer's disease. Journal of Clinical Gerontology and Geriatrics, 4: 33-6.

Cui X, Bray S, Bryant DM, et al (2011) A quantitative comparison of NIRS and $\mathrm{fMRI}$ across multiple cognitive tasks. Neuroimage, 54: 2808-21.

Dempsey JP, Harris KS, Shumway ST, et al (2015) Functional near infrared spectroscopy as a potential biological assessment of addiction recovery: preliminary findings. American Journal of Drug and Alcohol Abuse, 41: 119-26.

Ehlis AC, Herrmann MJ, Plichta MM, et al (2007) Cortical activation during two verbal fluency tasks in schizophrenic patients and healthy controls as assessed by multi-channel near-infrared spectroscopy. Psychiatry Research, 156: 1-13.

Ehlis AC, Bähne CG, Jacob CP, et al (2008) Reduced lateral prefrontal activation in adult patients with attention-deficit/hyperactivity disorder (ADHD) during a working memory task: a functional near-infrared spectroscopy (fNIRS) study. Journal of Psychiatric Research, 42: 1060-7.

Fekete T, Beacher FDCC, Cha J, et al (2014) Small-world network properties in prefrontal cortex correlate with predictors of psychopathology risk in young children: a NIRS study. Neuroimage, 85: 345-53.

Ferrari M, Quaresima V (2012) A brief review on the history of human functional near-infrared spectroscopy (fNIRS) development and fields of application. Neuroimage, 63: 921-35

Fukuda M (2015) fNIRS as an auxiliary laboratory test for differential diagnosis of depressive state: clinical application of near-infrared spectroscopy (NIRS) as the first trial for approved laboratory tests in psychiatry. Seishin Shinkeigaku Zasshi, 117: 79-93.

Fukuda M, Mikuni M (2012) Clinical application of near-infrared spectroscopy (NIRS) in psychiatry: the advanced medical technology for differential diagnosis of depressive state. Seishin Shinkeigaku Zasshi, 114: 801-6.

Ho CSH, Zhang MWB, Ho RCM (2016) Optical topography psychiatry: a chip off the old block or a new look beyond the mind-brain frontiers? Frontiers in Psychiatry, 7: 74.

Hoshi Y (2016) Hemodynamic signals in fNIRS. In New Horizons in Neurovascular Coupling: A Bridge Between Brain Circulation and Neural Plasticity (eds K Masamoto, H Hirase, K Yamada): 153-82. Elsevier.

Inoue Y, Sakihara K, Gunji A, et al (2012) Reduced prefrontal hemodynamic response in children with ADHD during the Go/NoGo task: a NIRS study. NeuroReport, 23: 55-60.

Ishii-Takahashi A, Takizawa R, Nishimura Y, et al (2014) Prefrontal activation during inhibitory control measured by near-infrared spectroscopy for differentiating between autism spectrum disorders and attention deficit hyperactivity disorder in adults. Neurolmage, 4: 53-63.

Koike S, Nishimura Y, Takizawa R, et al (2013) Near-infrared spectroscopy in schizophrenia: a possible biomarker for predicting clinical outcome and treatment response. Frontiers in Psychiatry, 4: 145.

Kubota Y, Toichi M, Shimizu M, et al (2005) Prefrontal activation during verbal fluency tests in schizophrenia: a near-infrared spectroscopy (NIRS) study. Schizophrenia Research, 77: 65-73.

Jung CE, Strother L, Feil-Seifer DJ, et al (2016) Atypical asymmetry for processing human and robot faces in autism revealed by fNIRS. PLOS ONE, 11: e0158804

Liu XM, Sun GX, Zhang XO, et al (2014) Relationship between the prefrontal function and the severity of the emotional symptoms during a verbal fluency task in patients with major depressive disorder: a multi-channel NIRS study. Progress in Neuro-Psychopharmacology and Biological Psychiatry, 54: 114-21.

Martinelli C, Shergill SS (2015) Everything you wanted to know about neuroimaging and psychiatry, but were afraid to ask. BJPsych Advances, 21: $251-60$.

Nishimura Y, Tanii H, Hara N, et al (2009) Relationship between the prefrontal function during a cognitive task and the severity of the symptoms in patients with panic disorder: a multi-channel NIRS study. Psychiatry Research, 172: 168-72.

Noda T, Yoshida S, Matsuda T, et al (2012) Frontal and right temporal activations correlate negatively with depression severity during verbal fluency task: a multi-channel near-infrared spectroscopy study. Journal of Psychiatic Research, 46: 905-12.

Okada, K, Ota T, lida J, et al (2013) Lower prefrontal activity in adults with obsessive-compulsive disorder as measured by near-infrared spectroscopy. Progress in Neuro-Psychopharmacology and Biological Psychiatry, 43: 7-13.
MCQ answers

$1 \mathrm{a} \quad 2 \mathrm{~b} \quad 3 \mathrm{~d} \quad 4 \mathrm{~b} \quad 5 \mathrm{e}$ 
Sato H, Yahata N, Funane T, et al (2013) A NIRS-fMRI investigation of prefrontal cortex activity during a working memory task. Neuroimage, 83: $158-73$.

Schroeter ML, Zysset S, Kupka T, et al (2002) Near-infrared spectroscopy can detect brain activity during a color-word matching Stroop task in an event-related design. Human Brain Mapping, 17: 61-71.

Takahashi T (2011) Influence of skin blood flow on near-infrared spectroscopy signals measured on the forehead during a verbal fluency task. Neurolmage, 57: 991-1002.

Takanori S, Haruhide H, Yasuhiro W (2011) Independent component analysis technique to remove skin blood flow artifacts in functional near-infrared spectroscopy signals. Paper presented at 21 st Annual Conference of the Japanese Neural Network Society (December, 2011). JNNS (http://jnns.org/conference/misc/camera_ready/P3-04.pdf).

Takizawa R, Fukuda M, Kawasaki S, et al (2014) Neuroimaging-aided differential diagnosis of the depressive state. Neurolmage, 85: 498507.

Yamanaka K, Tomioka H, Kawasaki S, et al (2014) Effect of parietal transcranial magnetic stimulation on spatial working memory in healthy elderly persons--comparison of near infrared spectroscopy for young and elderly. PLOS ONE, 14: e102306.

\section{MCOs}

Select the single best option for each question stem

1 fNIRS is unlikely to be affected by:

a body movement

b skin integrity

c skull thickness

d thick scalp hair

e none of the above.

2 The light used in fNIRS is:

a gamma radiation

b infrared light

c radio waves

d sunlight

e ultraviolet light.

\section{A limitation of fNIRS compared with $\mathrm{fMRI}$} is that it:

a is portable

b is more expensive

c has lower temporal resolution

$\mathrm{d}$ has lower spatial resolution

e cannot be combined with EEG devices.

4 fNIRS cannot be used to assess the:

a frontoparietal cortex

b hippocampus

c occipital lobe

d prefrontal cortex

e superficial cortex.
5 The correlation coefficient for fNIRS activity and $f M R I$ signal during the same cognitive task is in the range:
a $0.17-0.24$
b $0.27-0.34$
c $0.37-0.54$
d $0.47-0.74$
e $0.77-0.94$. 